\title{
The Physical and Geometrical Interpretation of Fractional Order Derivatives
}

\author{
Ali Karci \\ Department of Computer Engineering, Inonu University, Turkey
}

Copyright $(2015$ by authors, all rights reserved. Authors agree that this article remains permanently open access under the terms of the Creative Commons Attribution License 4.0 International License

\begin{abstract}
The fractional order derivative is a famous subject and it has been studied about three centuries. There are not any studies on the physical and geometrical interpretation of fractional order derivative. The aim of this study is to interpret the geometrical meaning of the fractional order derivatives of any function. We used a new definition for fractional order derivative to interpret it. At this aim, simple and easily understandable subjects distance, velocity and acceleration were used to depict the experimental results and interpretations. The important contribution of this paper is that when the order of fractional order derivative approaches to 1 , the result of derivative process approaches to classical derivative and operator is linear; otherwise, the result of derivative process is different from classical derivative and applied operator is non-linear.
\end{abstract}

Keywords Fractional Calculus, Fractional Order Derivatives, Mathematical Analysis

\section{Introduction}

The fractional calculus is an old research subject and there is a belief that fractional calculus may model physical systems more than classical calculus. The fractional calculus is used as fractional derivatives or integrals, and it is not a local point calculus. In order to make fractional calculus use by more science and engineering communities, fractional calculus needs a new definition and approach, and also the geometrical meaning of fractional order derivatives must be known by researchers.

There are many researchers deal with this subject such as fractional calculus [1], solving fractional differential equation with differential transform method [2], analytical schemes for fractional differential equations [3], fractional equation for oscillator [4], waves in Hirota equations [5], soliton rings in quantum semiconductor plasmas [6], geometrical meaning of chain rule in fractional calculus [7], fractional-order formulation of power and exponential distributions [8], fractional entropies [9], new class of analytic functions derived by fractional differential operator [10], approximating spectral Jacobi-collocation for fractional order integro-differential equations of Volterra type [11], viscosity solution of nonlinear time fractional equation [12], positive solutions for a class of nonlinear fractional differential equations [13], etc. These are some of researches on the fractional calculus and fractional variations.

These studies include similar approach for fractional order derivatives (FODs). This means that the order of derivative is not integer; in general, conventional calculus deals with derivative/integration of integer order, on the other hand, fractional calculus deals with any order differentiation and integration. There is no a clear reasoning for fractional order derivative/integration. Due to this case, Alexopoulos and Weinberg tried to describe the meaning of fractional order derivatives by using power-law. They asked the following questions: "what is the physical interpretation of the half derivative of distance: $\mathrm{d}^{0.5} \mathrm{~s}(\mathrm{t}) / \mathrm{dt}^{0.5}$ where $\mathrm{s}(\mathrm{t})$ stands for distance? They tried to support their interpretation on the distance, velocity and acceleration. Alexopoulos and Weinberg have used the definitions in the literature for fractional order derivative and definitions involve some deficiencies. Due to this case, the same physical related concepts will be used in this article by using the proposed method in Karc1's papers [14, 15, 16, 17].

The fractional order derivative methods based on the series expansion and pseudo-continuity have deficiencies. Due to this case, there is a need to redefine fractional order derivative. In this point of view, there were some studies on the re-definition of fractional order derivatives $[14,15,16$, 17] involve a new method for fractional order derivatives. The physical and geometrical interpretations of fractional order derivatives were illustrated analytically and experimental by using distance, velocity and acceleration concepts by using this definition.

This article is organized as follow. Section 2 describes new approach for fractional order derivative and interpretation of fractional order derivative. Section 3 illustrates the experimental results. Finally, Section 4 finalized this article. 


\section{New Approach for FODs and Its Interpretation}

The definitions for fractional order derivative include deficiencies, and due to this case there is a need to re-define the fractional order derivative concept. This re-definition was done in Karc1's paper [14, 15, 16, 17] by using indefinite limit and L'Hospital rule. The detail of this re-definition is as follow.

The meaning of derivative is the rate of change in the dependent variable versus the changes in the independent variables. Assume that $f(x): R \rightarrow R$ is a function and the equation of derivative can be considered as

$$
f^{(1)}(x)=f^{\prime}(x)=\lim _{h \rightarrow 0} \frac{f(x+h)-f(x)}{(x+h)-x}
$$

The order of derivation can be considered as non-integer. There will be two cases:

Case 1: If the order of derivative is $\alpha \in R$,

$$
f^{(\alpha)}(x)=\left(\lim _{h \rightarrow 0} \frac{f(x+h)-f(x)}{(x+h)-x}\right)^{\alpha}=\left(f^{\prime}(x)\right)^{\alpha}
$$

This yields the derivative to power (exponent) $\alpha$, and this is not a fractional order derivative.

Case 2: If the order of derivative is $\alpha \in R$, then the fractional order derivative is

$$
f^{(\alpha)}(x)=\lim _{h \rightarrow 0} \frac{f^{\alpha}(x+h)-f^{\alpha}(x)}{(x+h)^{\alpha}-x^{\alpha}}
$$

In the case of very small value of $h$, the above limit concluded in indefinite limit such as

$$
f^{(\alpha)}(x)=\lim _{h \rightarrow 0} \frac{f^{\alpha}(x+h)-f^{\alpha}(x)}{(x+h)^{\alpha}-x^{\alpha}}=\frac{0}{0}
$$

In this case, the method used for indefinite limit (such as L'Hospital method) can be used, and the FOD can be rephrased as follow.

$$
\begin{aligned}
& f^{(\alpha)}(x)=\lim _{h \rightarrow 0} L\left(\frac{f^{\alpha}(x+h)-f^{\alpha}(x)}{(x+h)^{\alpha}-x^{\alpha}}\right) \\
& =\lim _{h \rightarrow 0} \frac{\frac{d\left(f^{\alpha}(x+h)-f^{\alpha}(x)\right)}{d h}}{\frac{d\left((x+h)^{\alpha}-x^{\alpha}\right)}{d h}}
\end{aligned}
$$

The some properties of FOD can be explained in the following theorems. The other information about this definition can be found in $[14,15,16,17]$.

Theorem 1: Assume that $\alpha, \beta \in \mathrm{R}$. When $\alpha \neq \beta, \mathrm{f}^{(\alpha)}(\mathrm{x}) \neq \mathrm{f}^{(\beta)}(\mathrm{x})$ where $\mathrm{f}: \mathrm{R} \rightarrow \mathrm{R}$.

Proof: The derivatives terms can be expressed as follow.

$$
f^{(\alpha)}(x)=\left(\frac{f(x)}{x}\right)^{\alpha-1} \frac{d f(x)}{d x} \text { and }
$$

$$
\begin{gathered}
f^{(\beta)}(x)=\left(\frac{f(x)}{x}\right)^{\beta-1} \frac{d f(x)}{d x} . \\
\frac{f^{(\alpha)}(x)}{f^{(\beta)}(x)}=\frac{\left(\frac{f(x)}{x}\right)^{\alpha-1} \frac{d f(x)}{d x}}{\left(\frac{f(x)}{x}\right)^{\beta-1} \frac{d f(x)}{d x}}=\frac{\left(\frac{f(x)}{x}\right)^{\alpha-1}}{\left(\frac{f(x)}{x}\right)^{\beta-1}} \neq 1 \text { when } \alpha \neq \beta
\end{gathered}
$$

Theorem 2: Assume that $\alpha, \beta \in \mathrm{R}$ and $\mathrm{f}: \mathrm{R} \rightarrow \mathrm{R}$, then $\left(f^{(\alpha)}(x)\right)^{(\beta)}(x) \neq f^{(\alpha+\beta)}(x)$.

Proof: The derivatives terms can be expressed as follow.

$$
\begin{aligned}
& f^{(\alpha)}(x)=\left(\frac{f(x)}{x}\right)^{\alpha-1} \frac{d f(x)}{d x} \\
& \left(f^{(\alpha)}(x)\right)^{(\beta)}(x)=\left(\frac{\left(\frac{f(x)}{x}\right)^{\alpha-1} \frac{d f(x)}{d x}}{x}\right)^{\beta-1} \frac{d\left(\left(\frac{f(x)}{x}\right)^{\alpha-1} \frac{d f(x)}{d x}\right)}{d x} \\
& f^{(\alpha+\beta)}(x)=\left(\frac{f(x)}{x}\right)^{\alpha+\beta-1} \frac{d f(x)}{d x} . \\
& \frac{\left(f^{(\alpha)}(x)\right)^{(\beta)}(x)}{f^{(\alpha+\beta)}(x)} \\
& =\frac{\left(\frac{\left.\left(\frac{f(x)}{x}\right)^{\alpha-1} \frac{d f(x)}{d x}\right)^{\beta-1}}{x} \frac{d\left(\left(\frac{f(x)}{x}\right)^{\alpha-1} \frac{d f(x)}{d x}\right)}{d x}\right.}{\left(\frac{f(x)}{x}\right)^{\alpha+\beta-1} \frac{d f(x)}{d x}} \neq 1
\end{aligned}
$$

Theorem 3: The FOD of distance, whose order is 2, is not same as acceleration.

Proof: Assume that $\mathrm{X}(\mathrm{t})$ is distance, and the acceleration is $a(t)=\frac{d^{2} X(t)}{d t^{2}}$ and the FOD of order 2 is

$$
a^{(\alpha)}(t)=a^{(2)}(t)=\left(\frac{X(t)}{t}\right) \frac{d X(t)}{d t} \neq \frac{d^{2} X(t)}{d t^{2}}
$$

Theorem 4: Assume that $\alpha, \beta \in \mathrm{R}, \alpha+\beta=1$ and $\mathrm{X}(\mathrm{t})$ is distance, $\mathrm{V}(\mathrm{t})$ is velocity and $\mathrm{A}(\mathrm{t})$ is acceleration. The following facts exist for FODs.
a) $\mathrm{X}^{(\alpha+\beta)}(\mathrm{x})=\mathrm{V}(\mathrm{t})$.
b) $\left(X^{(\alpha)}(t)\right)^{(\beta)}(t) \neq V(t)$.
c) $\quad\left(V^{(\alpha)}(t)\right)^{(\beta)}(t) \neq A(t)$
d) $\mathrm{X}^{(2)}(\mathrm{t}) \neq \mathrm{A}(\mathrm{t})$.
e) $\mathrm{V}^{(\alpha+\beta)}(\mathrm{t})=\mathrm{A}(\mathrm{t})$. 
Proof: The verifications of these equations can be illustrated by using Eq.1.

a) $X^{(\alpha+\beta)}(t)=\left(\frac{X(t)}{t}\right)^{\alpha+\beta-1} \frac{d X(t)}{d t}=\frac{d X(t)}{d t}=V(t)$

b) $\left(X^{(\alpha)}(t)\right)^{(\beta)}$

$=\left(\left(\frac{X(t)}{t}\right)^{\alpha-1} \frac{d X(t)}{d t}\right)^{\beta-1} \frac{d\left(\left(\frac{X(t)}{t}\right)^{\alpha-1} \frac{d X(t)}{d t}\right)}{d t}$

$\neq V(t)$

c)

$$
\begin{aligned}
& \left(V^{(\alpha)}(t)\right)^{(\beta)} \\
& =\left(\left(\frac{V(t)}{t}\right)^{\alpha-1} \frac{d V(t)}{d t}\right)^{\beta-1} \frac{d\left(\left(\frac{V(t)}{t}\right)^{\alpha-1} \frac{d V(t)}{d t}\right)}{d t} \neq A(t)
\end{aligned}
$$

d) $X^{(2)}(t)=\left(\frac{X(t)}{t}\right)^{2-1} \frac{d X(t)}{d t}=\frac{X(t)}{t} \frac{d X(t)}{d t} \neq A(t)$

e) $\quad V^{(\alpha+\beta)}(t)=\left(\frac{V(t)}{t}\right)^{\alpha+\beta-1} \frac{d V(t)}{d t}=\frac{d V(t)}{d t}=A(t)$

Theorem 5: Assume that $\mathrm{f}: \mathrm{R} \rightarrow \mathrm{R}, \alpha \in \mathrm{R}$, and $\mathrm{x}_{0}, \mathrm{x}_{1}$ are two extreme points. The following conditions are satisfied.

a) If $\mathrm{f}\left(\mathrm{x}_{0}\right)$ is maximum, $\mathrm{f}\left(\mathrm{x}_{1}\right)$ is minimum and $\mathrm{x}_{\mathrm{a}} \in\left(\mathrm{x}_{0}, \mathrm{x}_{1}\right)$ is inflection point, then $\forall \mathrm{x}_{\mathrm{b}} \in\left(\mathrm{x}_{0}, \mathrm{x}_{1}\right), \mathrm{f}^{(\alpha)}\left(\mathrm{x}_{\mathrm{a}}\right) \leq \mathrm{f}^{(\alpha)}\left(\mathrm{x}_{\mathrm{b}}\right)$.

b) If $\mathrm{f}\left(\mathrm{x}_{0}\right)$ is minimum, $\mathrm{f}\left(\mathrm{x}_{1}\right)$ is maximum and $\mathrm{x}_{\mathrm{a}} \in\left(\mathrm{x}_{0}, \mathrm{x}_{1}\right)$ is inflection point, then $\forall \mathrm{x}_{\mathrm{b}} \in\left(\mathrm{x}_{0}, \mathrm{x}_{1}\right), \mathrm{f}^{(\alpha)}\left(\mathrm{x}_{\mathrm{a}}\right) \geq \mathrm{f}^{(\alpha)}\left(\mathrm{x}_{\mathrm{b}}\right)$.

Proof: Let $\alpha, \varepsilon \in \mathrm{R}$ and $\varepsilon<<1$.

a) Assume that the order of derivation is $\alpha$. Then

$$
\begin{gathered}
f^{(\alpha)}(x)=\lim _{h \rightarrow 0} \frac{f^{\alpha}(x+h)-f^{\alpha}(x)}{(x+h)^{\alpha}-x^{\alpha}} \\
\lim _{\varepsilon \rightarrow 0} \frac{f^{\alpha}\left(x_{0}+\varepsilon\right)-f^{\alpha}\left(x_{0}\right)}{\left(x_{0}+\varepsilon\right)^{\alpha}-x_{0}^{\alpha}} \\
\geq \lim _{\varepsilon \rightarrow 0} \frac{f^{\alpha}\left(x_{0}+2 \varepsilon\right)-f^{\alpha}\left(x_{0}\right)}{\left(x_{0}+2 \varepsilon\right)^{\alpha}-x_{0}^{\alpha}} \\
\lim _{\varepsilon \rightarrow 0} \frac{f^{\alpha}\left(x_{0}+2 \varepsilon\right)-f^{\alpha}\left(x_{0}\right)}{\left(x_{0}+2 \varepsilon\right)^{\alpha}-x_{0}^{\alpha}} \\
\geq \lim _{\varepsilon \rightarrow 0} \frac{f^{\alpha}\left(x_{0}+3 \varepsilon\right)-f^{\alpha}\left(x_{0}\right)}{\left(x_{0}+3 \varepsilon\right)^{\alpha}-x_{0}^{\alpha}} \\
\lim _{\varepsilon \rightarrow 0} \frac{f^{\alpha}\left(x_{0}+(n-1) \varepsilon\right)-f^{\alpha}\left(x_{0}\right)}{\left(x_{0}+(n-1) \varepsilon\right)^{\alpha}-x_{0}^{\alpha}} \\
\lim _{\varepsilon \rightarrow 0} \frac{f^{\alpha}\left(x_{0}+n \varepsilon\right)-f^{\alpha}\left(x_{0}\right)}{\left(x_{0}+n \varepsilon\right)^{\alpha}-x_{0}^{\alpha}}
\end{gathered}
$$

and $\mathrm{x}+\mathrm{n} \varepsilon=\mathrm{x}_{\mathrm{a}}, \mathrm{x}_{\mathrm{b}}=\mathrm{x}_{0}+(\mathrm{n}-1) \varepsilon$, and $\mathrm{n} \in \mathrm{Z}^{+}$. The same reasoning is valid for interval $\left(\mathrm{x}_{\mathrm{a}}, \mathrm{x}_{1}\right)$ in reverse order.

b) Assume that the order of derivation is $\alpha$.

$$
\begin{aligned}
& \lim _{\varepsilon \rightarrow 0} \frac{f^{\alpha}\left(x_{0}+\varepsilon\right)-f^{\alpha}\left(x_{0}\right)}{\left(x_{0}+\varepsilon\right)^{\alpha}-x_{0}^{\alpha}} \\
& \leq \lim _{\varepsilon \rightarrow 0} \frac{f^{\alpha}\left(x_{0}+2 \varepsilon\right)-f^{\alpha}\left(x_{0}\right)}{\left(x_{0}+2 \varepsilon\right)^{\alpha}-x_{0}^{\alpha}} \\
& \quad \text { where } \mathrm{x}_{\mathrm{b}}=\mathrm{x}_{0}+\varepsilon . \\
& \lim _{\varepsilon \rightarrow 0} \frac{f^{\alpha}\left(x_{0}+2 \varepsilon\right)-f^{\alpha}\left(x_{0}\right)}{\left(x_{0}+2 \varepsilon\right)^{\alpha}-x_{0}^{\alpha}} \\
& \leq \lim _{\varepsilon \rightarrow 0} \frac{f^{\alpha}\left(x_{0}+3 \varepsilon\right)-f^{\alpha}\left(x_{0}\right)}{\left(x_{0}+3 \varepsilon\right)^{\alpha}-x_{0}^{\alpha}}
\end{aligned}
$$

c)

$$
\begin{aligned}
& \lim _{\varepsilon \rightarrow 0} \frac{f^{\alpha}\left(x_{0}+(n-1) \varepsilon\right)-f^{\alpha}\left(x_{0}\right)}{\left(x_{0}+(n-1) \varepsilon\right)^{\alpha}-x_{0}^{\alpha}} \\
& \leq \lim _{\varepsilon \rightarrow 0} \frac{f^{\alpha}\left(x_{0}+n \varepsilon\right)-f^{\alpha}\left(x_{0}\right)}{\left(x_{0}+n \varepsilon\right)^{\alpha}-x_{0}^{\alpha}}
\end{aligned}
$$

and $\mathrm{x}+\mathrm{n} \varepsilon=\mathrm{x}_{\mathrm{a}}, \mathrm{x}_{\mathrm{b}}=\mathrm{x}_{0}+(\mathrm{n}-1) \varepsilon$, and $\mathrm{n} \in \mathrm{Z}^{+}$. The same reasoning is valid for interval $\left(\mathrm{x}_{\mathrm{a}}, \mathrm{x}_{1}\right)$ in reverse order

\section{Experimental Results of FODs}

Alexopoulos and Weinberg tried to explain the meaning of fractional order derivatives by using distance, velocity and acceleration equations. When the order of derivative is 0.5 for distance equation, this mean is not summing the $50 \%$ of distance and $50 \%$ of velocity. The aim of this paper is to demonstrate the meaning of fractional order derivatives on the equations of distance, velocity and acceleration.

\subsection{Velocity vs Distance with Constant Acceleration}

Assume that acceleration is $4(\mathrm{a}=4)$. Then the equation of distance in this case is $\mathrm{X}(\mathrm{t})=\mathrm{V}_{0} \mathrm{t}+2 \mathrm{t}^{2}$ with time interval $t \in[0,10]$ and velocity equation can be obtained by taking first derivative such as

$$
\begin{gathered}
V(t)=\frac{d X(t)}{d t}=\frac{d\left(V_{0} t+2 t^{2}\right)}{d t}=V_{0}+4 t \text { with initial velocity } \\
\mathrm{V}_{0}=10 .
\end{gathered}
$$

The first derivative (velocity) of distance is seen in Figure 1 as blue and thick line. The fractional order derivative (FOD) of $\mathrm{X}(\mathrm{t})$ by using equation (1) is

$$
\begin{aligned}
& V^{(\alpha)}(t)=\left(\frac{X(t)}{t}\right)^{\alpha-1} \frac{d X(t)}{d t} \\
& =\left(\frac{10 t+2 t^{2}}{t}\right)^{\alpha-1}(10+4 t)=(10+2 t)^{\alpha-1}(10+4 t)
\end{aligned}
$$

where $\alpha$ is order of derivative and $\alpha \in \mathrm{R}$. Figure 1 illustrates the FODs of $\mathrm{X}(\mathrm{t})$ for order list such as $\{0.1,0.2,0.3,0.4,0.5$, 
$0.6,0.7,0.8,0.9,1,1.1,1.2,1.3,1.4,1.5,1.6,1.7,1.8,1.9,2\}$. The important point here is the coincidence of first derivative of $X(t)$ and FOD of $X(t)$ with order 1 are equal. The FOD of $\mathrm{X}(\mathrm{t})$ is seen in Figure 1 as cyan line where it coincides on the blue thick line (first derivative of $\mathrm{X}(\mathrm{t})$ ). When the order of derivative is greater than 1 , the result is greater than first derivative for monotonic increasing functions (Figure 1). The velocity is the first derivative of distance with respect to time variable $t$. The abbreviation F.Vel.a $=0.3$ stands for fractional order derivative of distance with alpha value 0.3 . When the order of derivative is approaching to 1 , fractional order derivative approaches to velocity for derivative of distance. When order of derivative diverges from 1 , the result diverges from the velocity and its geometrical meaning

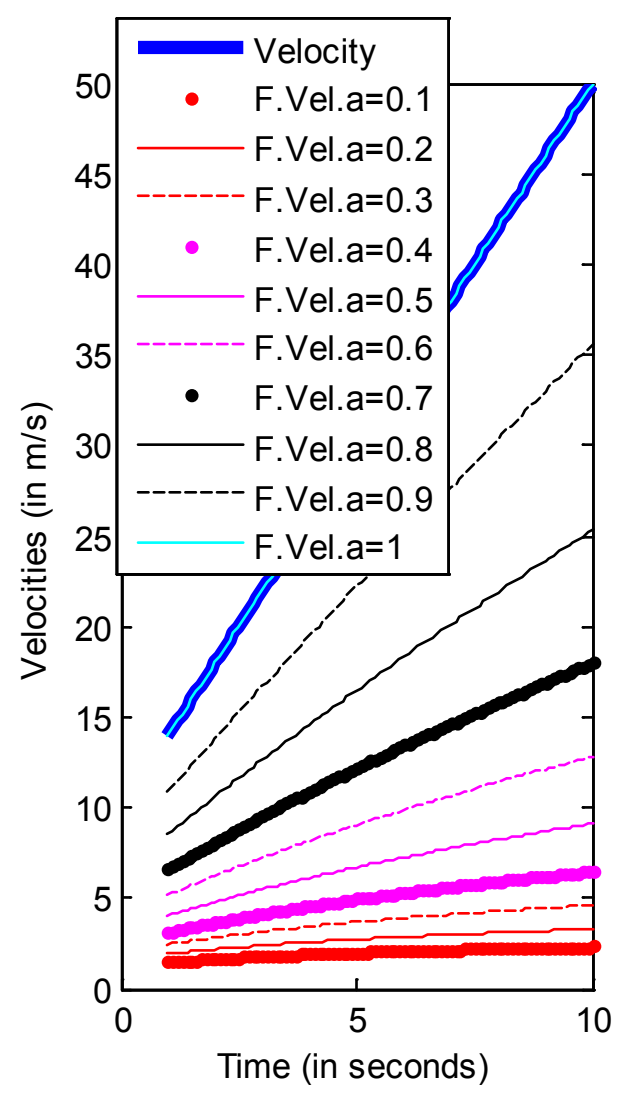

is not known clearly for other values of order.

\subsection{Velocity vs Distance with Variable Acceleration}

Assume that acceleration is $3 \mathrm{t}$ with $\mathrm{t} \in[0,10] \quad(\mathrm{a}(\mathrm{t})=3 \mathrm{t})$. Then the equation of velocity in this case is

$$
V(t)=\int_{0}^{t} 3 \eta d \eta=\frac{3}{2} t^{2}+V_{0} \text { with initial velocity } \mathrm{V}_{0}=10 .
$$

and the distance equation is

$$
X(t)=\int_{0}^{t}\left(\frac{3}{2} \eta^{2}+V_{0}\right) d \eta=\frac{1}{2} t^{3}+V_{0} t
$$

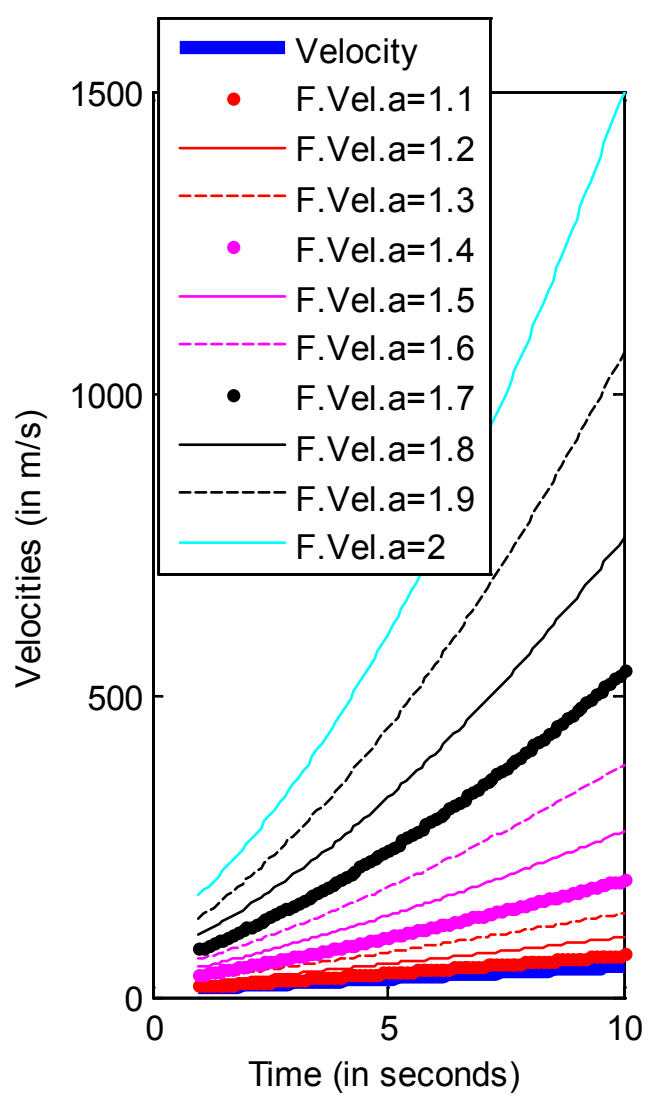

Figure 1. The fractional order derivatives of distance for orders $=\{0.1,0.2,0.3,0.4,0.5,0.6,0.7,0.8,0.9,1,1.1,1.2,1.3,1.4,1.5,1.6,1.7,1.8,1.9,2\}$ (acceleration is constant). 
The velocity equation can be obtained by taking first derivative such as

$$
V(t)=\frac{d X(t)}{d t}=\frac{d\left(V_{0} t+2 t^{2}\right)}{d t}=V_{0}+4 t
$$

The first derivative (velocity) of distance is seen in Figure 2 as blue and thick line. The FOD of $\mathrm{X}(\mathrm{t})$ by using equation (1) is

$$
\begin{aligned}
& V^{(\alpha)}(t)=\left(\frac{X(t)}{t}\right)^{\alpha-1} \frac{d X(t)}{d t}=\left(\frac{10 t+(1 / 2) t^{3}}{t}\right)^{\alpha-1}\left(10+\frac{3}{2} t^{2}\right) \\
& =\left(10+\frac{1}{2} t^{2}\right)^{\alpha-1}\left(10+\frac{3}{2} t^{2}\right)
\end{aligned}
$$

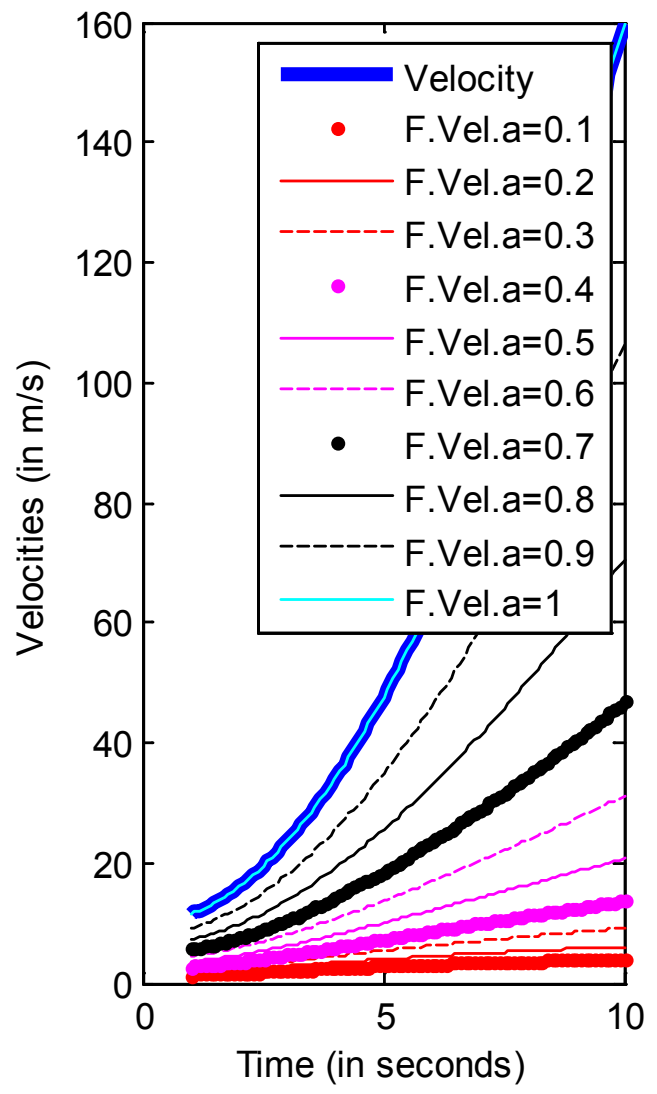

where $\alpha \in \mathrm{R}$. Figure 2 illustrates the FODs of $\mathrm{X}(\mathrm{t})$ for order list such as $\{0.1,0.2,0.3,0.4,0.5,0.6,0.7,0.8,0.9,1,1.1,1.2$, $1.3,1.4,1.5,1.6,1.7,1.8,1.9,2\}$. The important point here is the coincidence of first derivative of $X(t)$ and FOD of $X(t)$ with order 1 are equal. The FOD of $X(t)$ is seen in Figure 2 as cyan line where it coincides on the blue thick line (first derivative of $\mathrm{X}(\mathrm{t})$ ). When the order of derivative is greater than 1 , the result is greater than first derivative for monotonic increasing functions (Figure 2). The velocity is the first derivative of distance with respect to time variable $t$. When the acceleration is variable, the meanings of derivations stand same. The order of derivative converges to 1,FOD of distance converges to velocity. When order of derivative diverges from 1 , the result diverges from the velocity and its geometrical meaning is not known clearly for other values of FOD order.

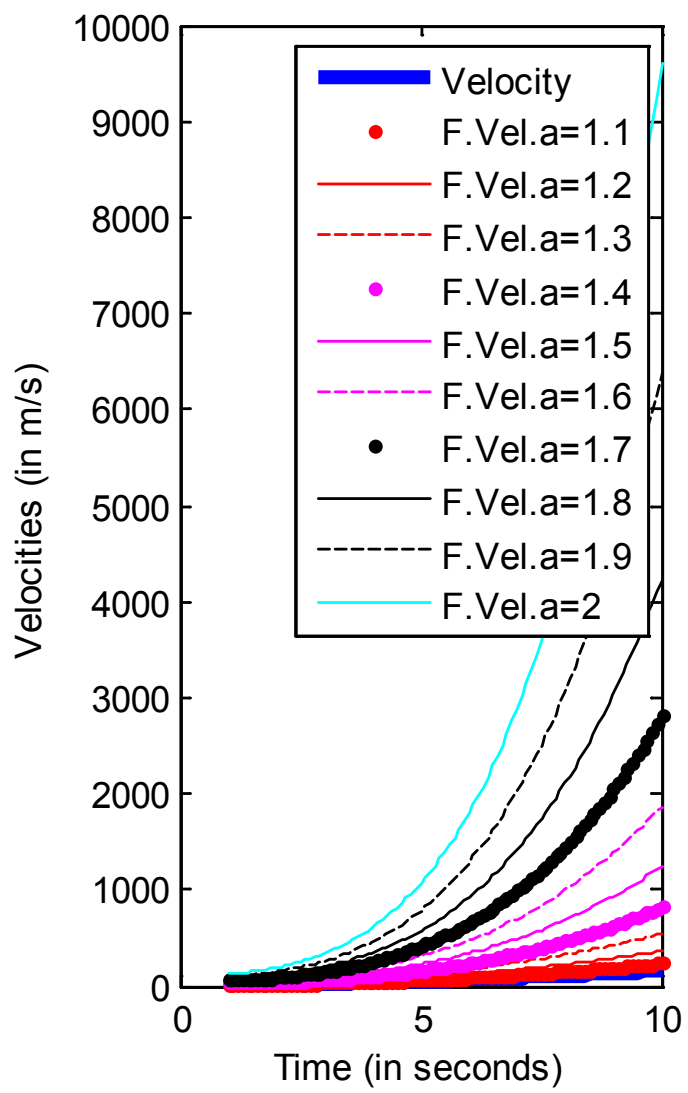

Figure 2. The fractional order derivatives of distance for orders $=\{0.1,0.2,0.3,0.4,0.5,0.6,0.7,0.8,0.9,1,1.1,1.2,1.3,1.4,1.5,1.6,1.7,1.8,1.9,2\}$ (acceleration is variable). 


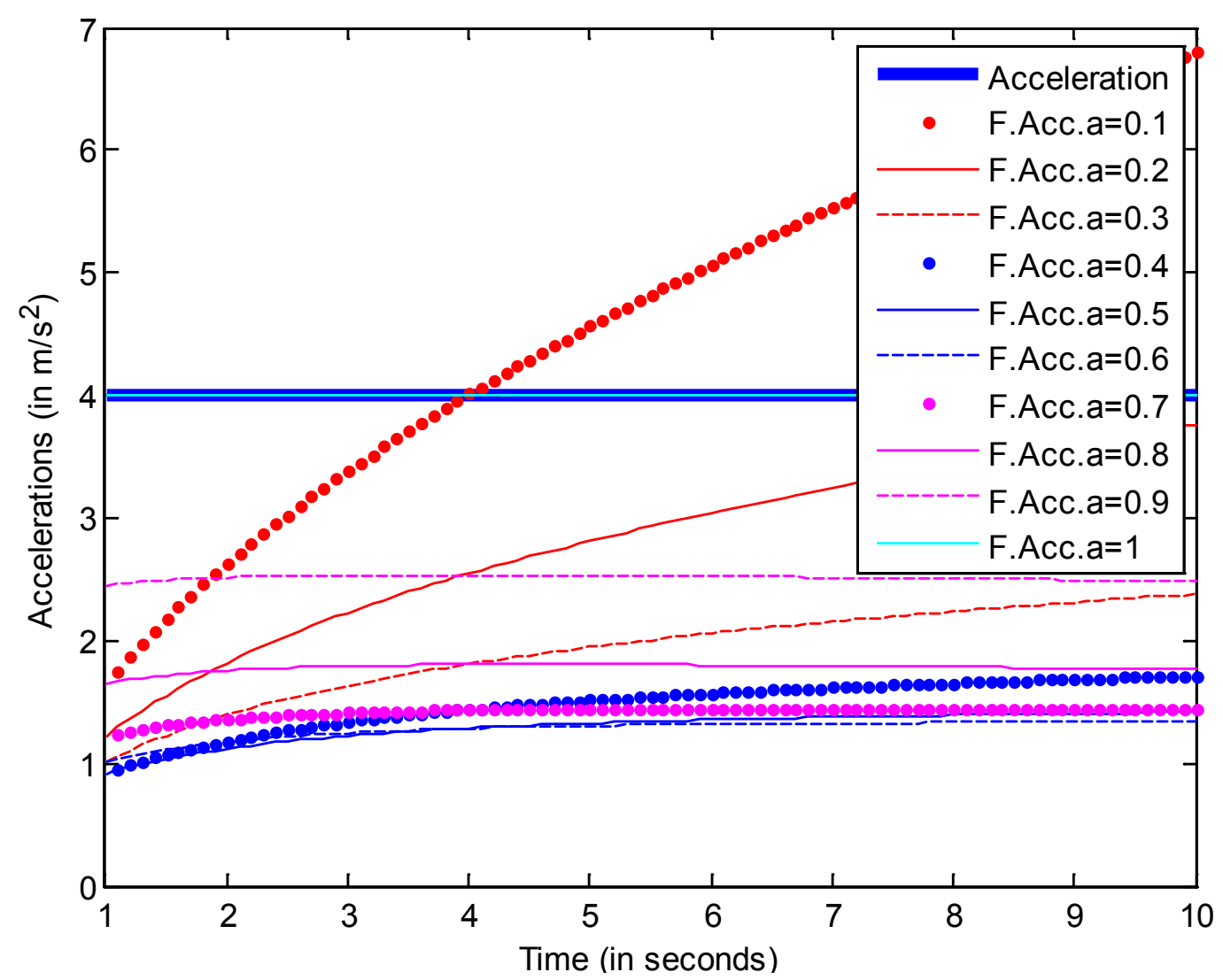

Figure 3. The fractional order derivatives of velocity for orders $=\{0.1,0.2,0.3,0.4,0.5,0.6,0.7,0.8,0.9,1\}$ (acceleration is constant).

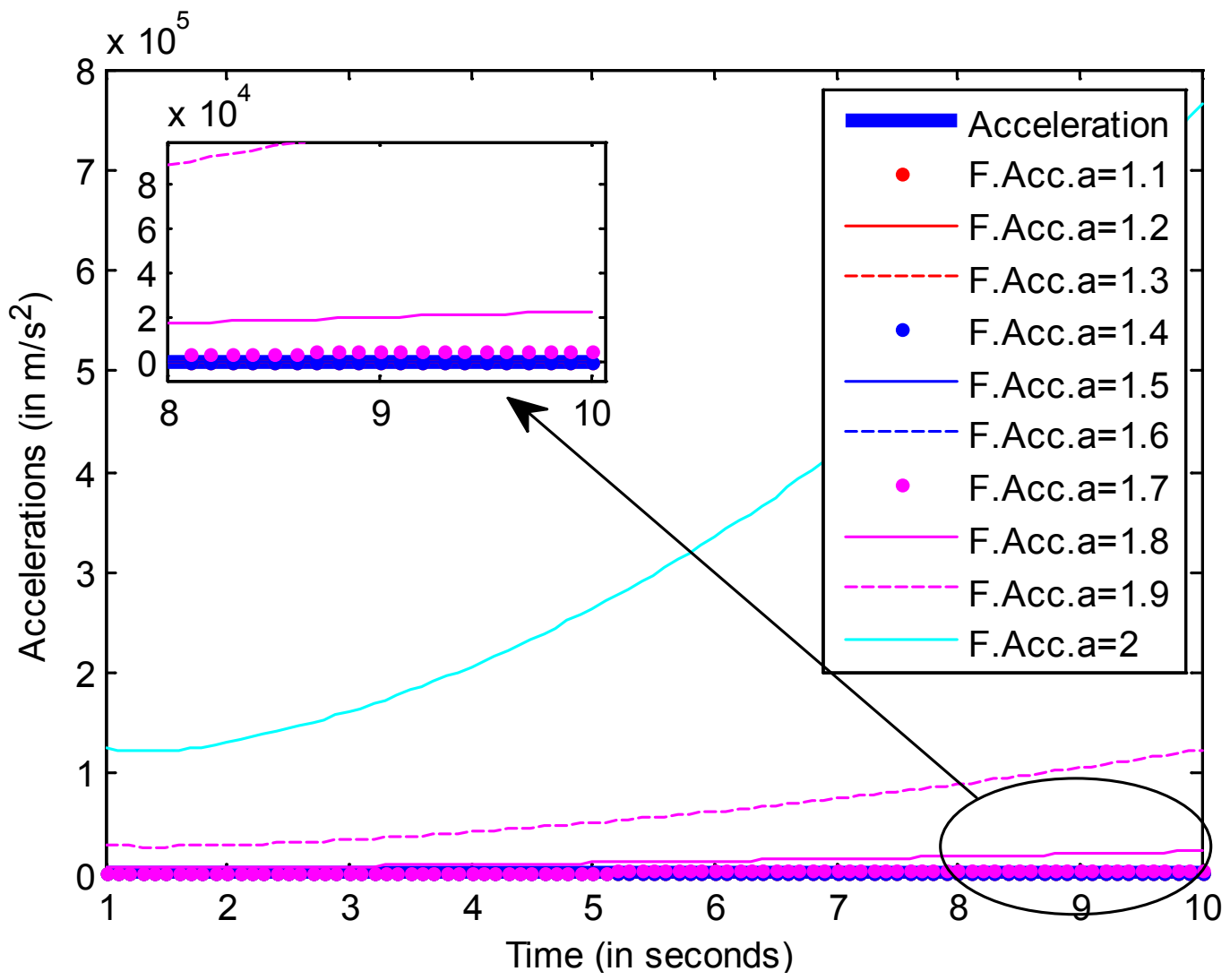

Figure 4. The fractional order derivatives of distance for orders $=\{1.1,1.2,1.3,1.4,1.5,1.6,1.7,1.8,1.9,2\}$ (acceleration is constant). 


\subsection{Constant Acceleration vs Velocity}

There will be two cases for accelerations: Constant acceleration and variable acceleration. When acceleration is constant, the FODs of velocity can be compared to constant acceleration. Assume that acceleration is $4(\mathrm{a}=4)$. Then the equation of velocity in this case is $\mathrm{V}(\mathrm{t})=\mathrm{V}_{0}+4 \mathrm{t}$ with time interval $t \in[0,10]$ and initial velocity $V_{0}=10$. FODs of velocity is

$$
\begin{aligned}
& a^{(\alpha)}(t)=\left(\frac{(10+2 t)^{\alpha-1}(10+4 t)}{t}\right)^{\alpha-1} \\
& {\left[(\alpha-1)(10+2 t)^{\alpha-2}(10+4 t)+4(10+2 t)^{\alpha-1}\right]}
\end{aligned}
$$

The first derivative (acceleration) of velocity is seen in Figure 3 as blue and thick line. The FODs of $\mathrm{V}(\mathrm{t})$ by using equation (1) are also seen in Figure 3, and it can be seen easily that when order of derivative of velocity approaches to 1 , the FOD converges to acceleration. On contrary, when order of derivative gets away from 1, the FOD diverges from the acceleration (Figure 4) and the geometrical meanings of obtained results are not known clearly.

\subsection{Variable Acceleration vs Velocity}

The case of variable acceleration will be explained in this section. When acceleration is variable, the FODs of velocity can be compared to variable acceleration. Assume that acceleration is $3 \mathrm{t}(\mathrm{a}(\mathrm{t})=3 \mathrm{t})$. Then the equation of velocity in this case is $V(t)=\int_{0}^{t} 3 \eta d \eta=\frac{3}{2} t^{2}+V_{0}$ with time interval $\mathrm{t} \in[0,10]$ and initial velocity $\mathrm{V}_{0}=10$. FOD of velocity is

$$
\begin{aligned}
& a^{(\alpha)}(t)=\left[\frac{\left(10+\frac{1}{2} t^{2}\right)^{\alpha-1}\left(10+\frac{3}{2} t^{2}\right)}{t}\right]^{\alpha-1} \\
& {\left[(\alpha-1) t\left(10+\frac{1}{2} t^{2}\right)^{\alpha-2}\left(10+\frac{3}{2} t^{2}\right)+3 t\left(10+\frac{1}{2} t^{2}\right)^{\alpha-1}\right]}
\end{aligned}
$$

The first derivative (acceleration) of velocity is seen in Figure 5 as blue and thick line. The FODs of V(t) by using equation (1) are also seen in Figure 5 and Figure 6 , and it can be seen easily that when order of derivative of velocity approaches to 1 , the FOD converges to acceleration. On contrary, when order of derivative gets away from 1, the FOD diverges from the acceleration (Figure 6) and obtained results are not known clearly.

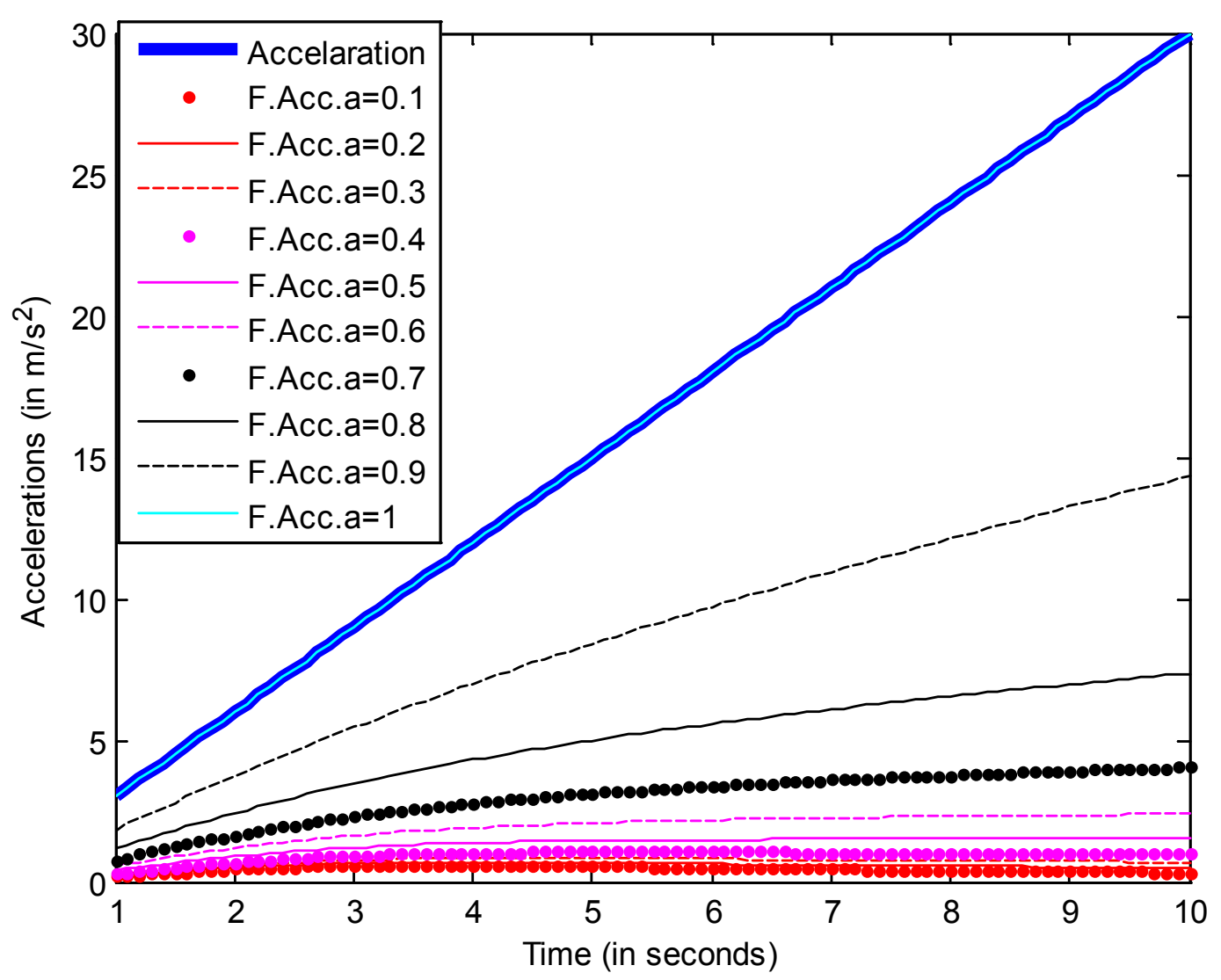

Figure 5. The fractional order derivatives of velocity for orders $=\{0.1,0.2,0.3,0.4,0.5,0.6,0.7,0.8,0.9,1\}$ (acceleration is variable). 


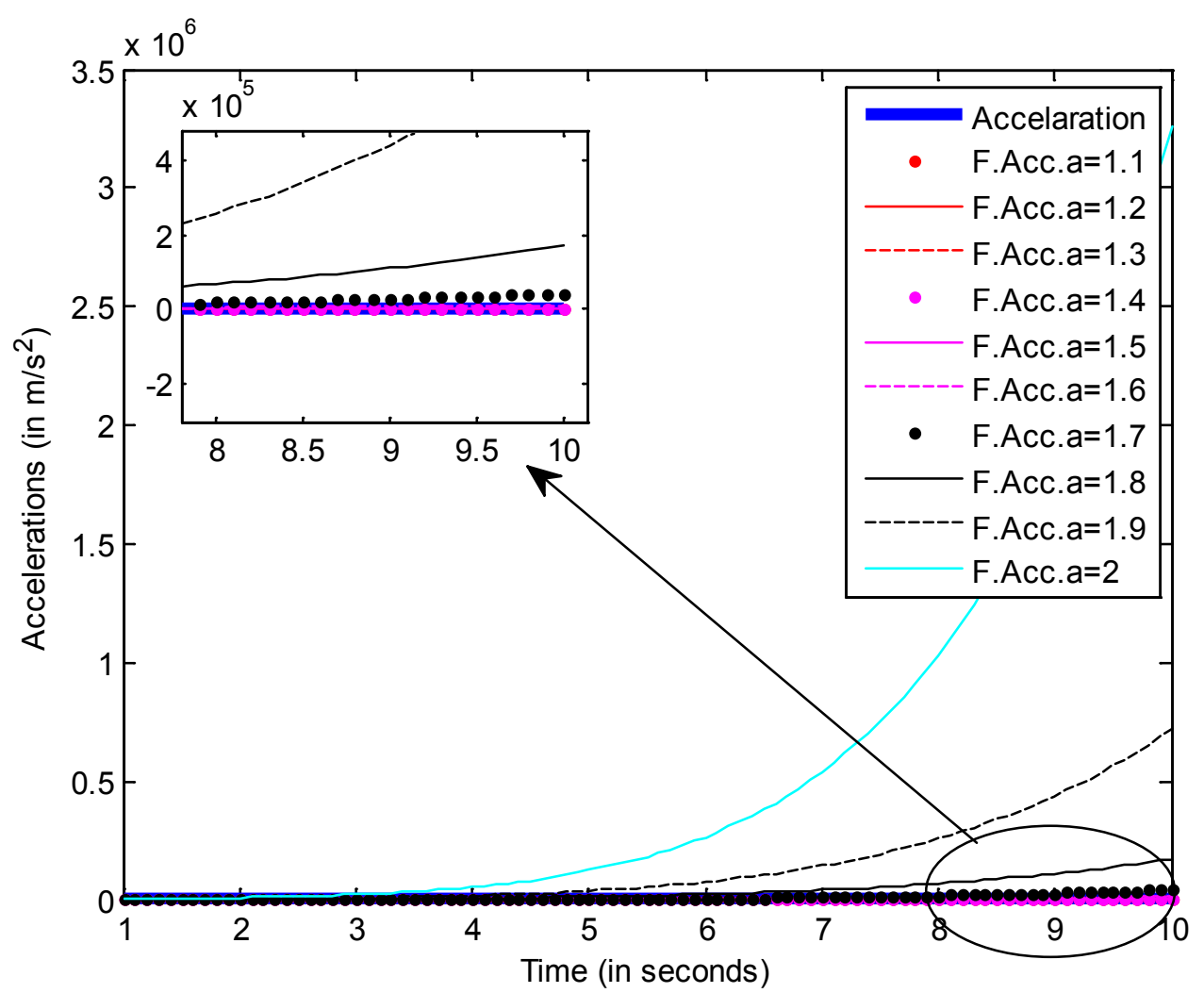

Figure 6. The fractional order derivatives of velocity for orders $=\{1.1,1.2,1.3,1.4,1.5,1.6,1.7,1.8,1.9,2\}$ (acceleration is variable).

\subsection{Velocity and FODs of Distance for Constant Acceleration}

The effect of order on the derivation can be illustrated on a small deviation in derivation orders. Figure 7 and Figure 8 depict that FOD gets closer to the first derivation of distance with constant acceleration.

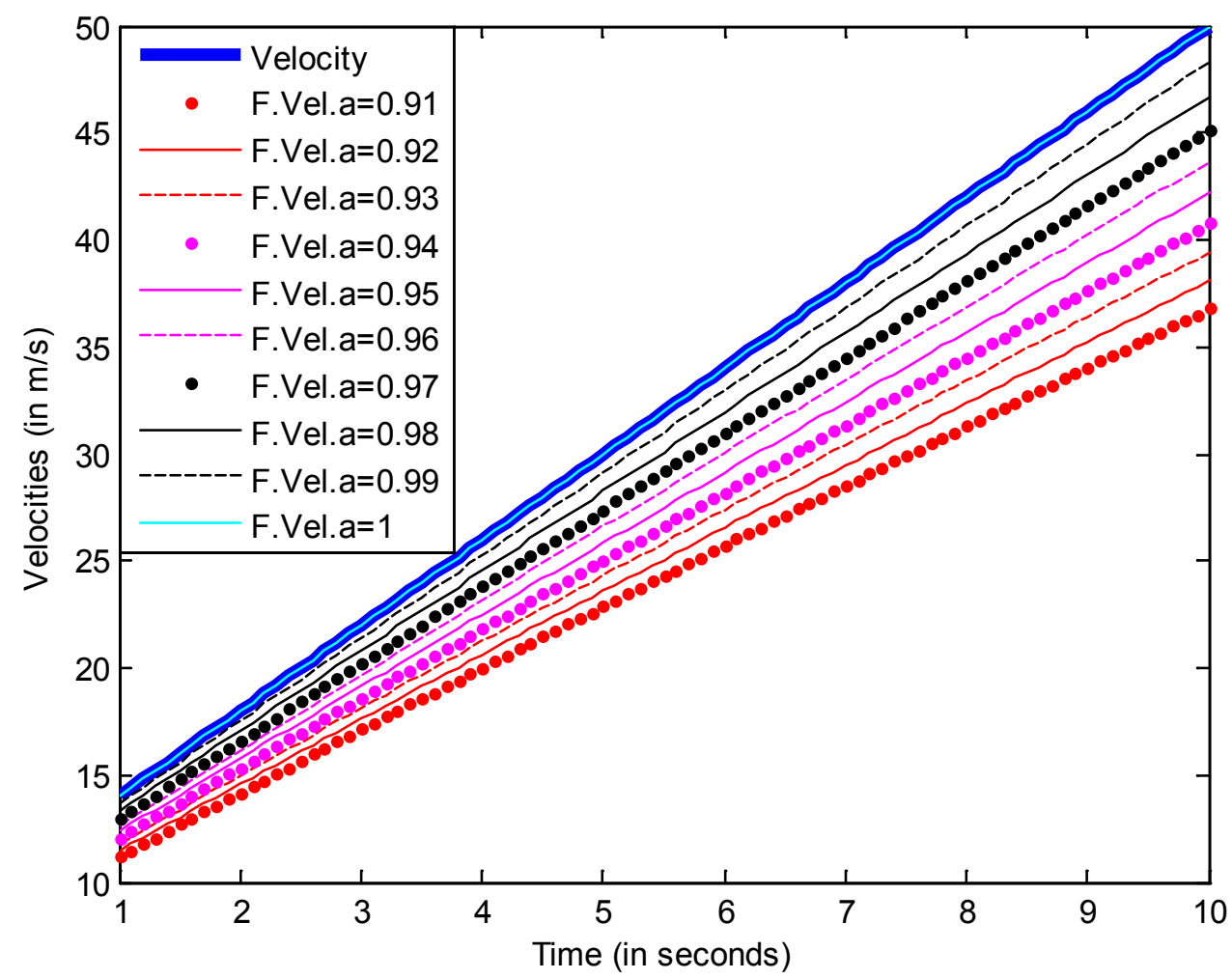

Figure 7. The fractional order derivatives of velocity for orders $=\{0.1,0.2,0.3,0.4,0.5,0.6,0.7,0.8,0.9,1\}$ (acceleration is constant). 


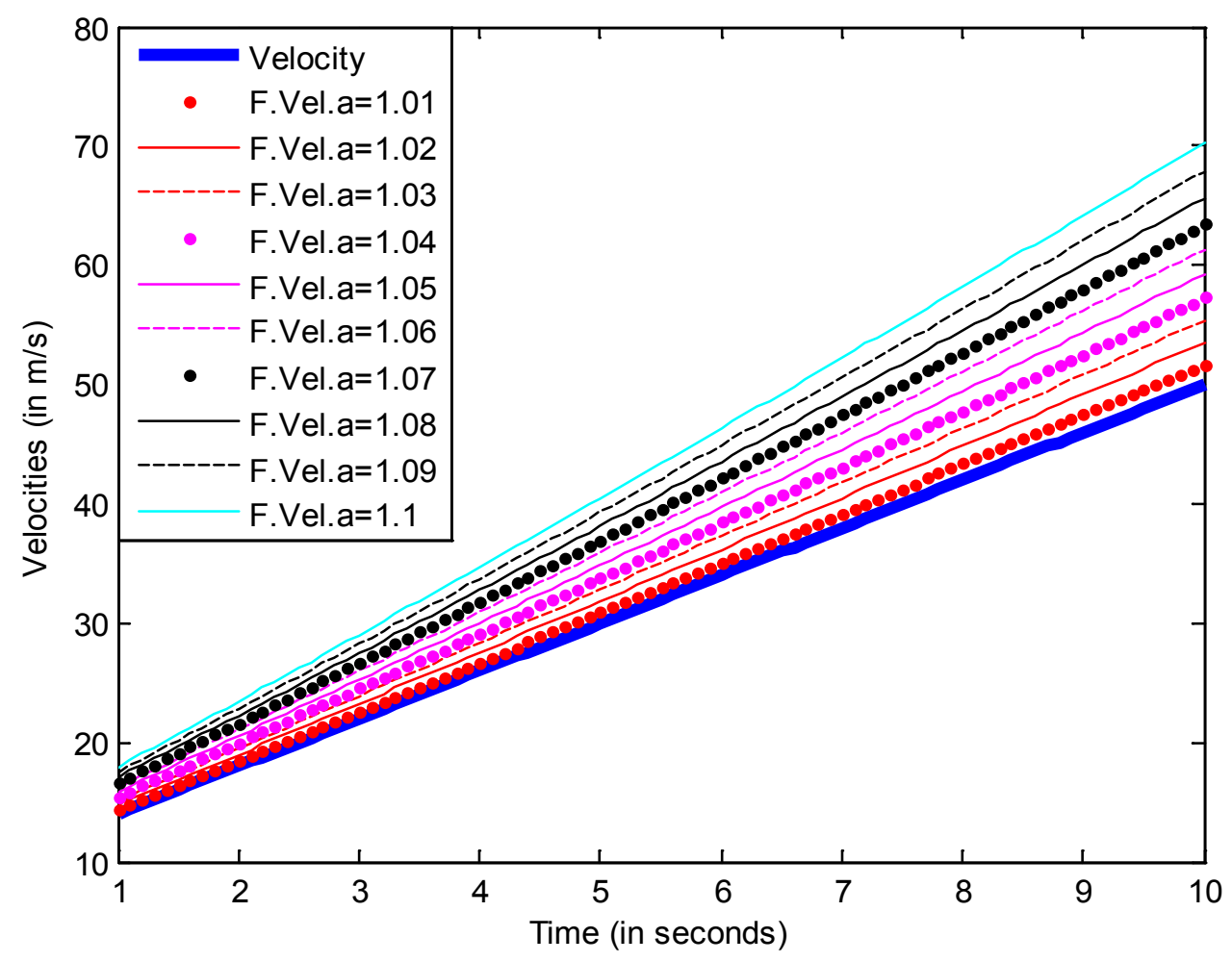

Figure 8. The fractional order derivatives of velocity for orders $=\{1.1,1.2,1.3,1.4,1.5,1.6,1.7,1.8,1.9,2\}$ (acceleration is constant).

\subsection{Velocity and FODs of Distance for Variable Acceleration}

The effect of order on the derivation can be illustrated on a small deviation in derivation orders. Figure 9 and Figure 10 depicts that FOD gets closer to the first derivation of distance with constant acceleration. When order of derivation gets away from 1, the obtained results will be different from the first derivative of distance (velocity); on the other hand, when order of derivation converges to 1 , the obtained results get more similar to first derivative of distance (velocity).

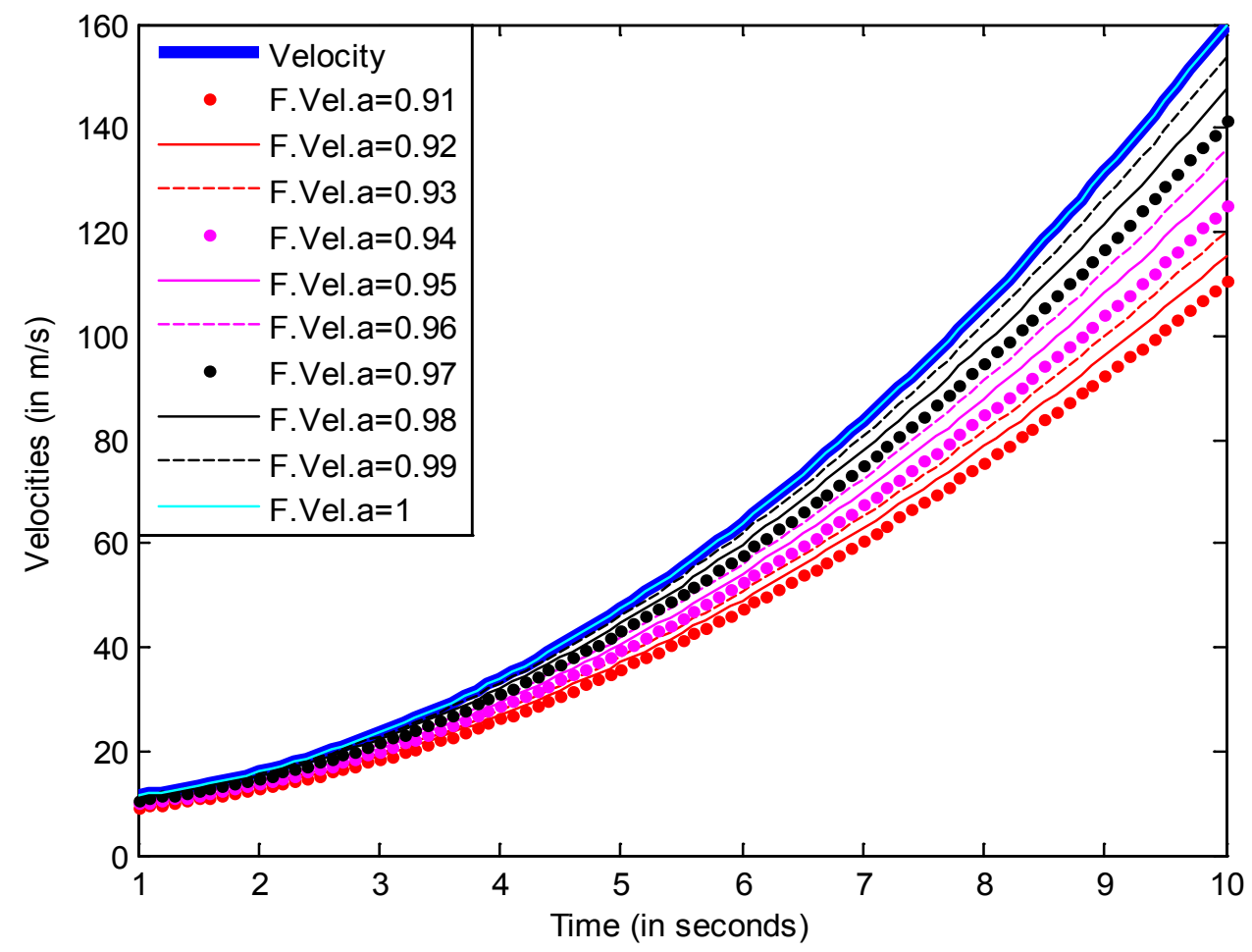

Figure 9. The fractional order derivatives of velocity for orders $=\{0.1,0.2,0.3,0.4,0.5,0.6,0.7,0.8,0.9,1\}$ (acceleration is variable). 


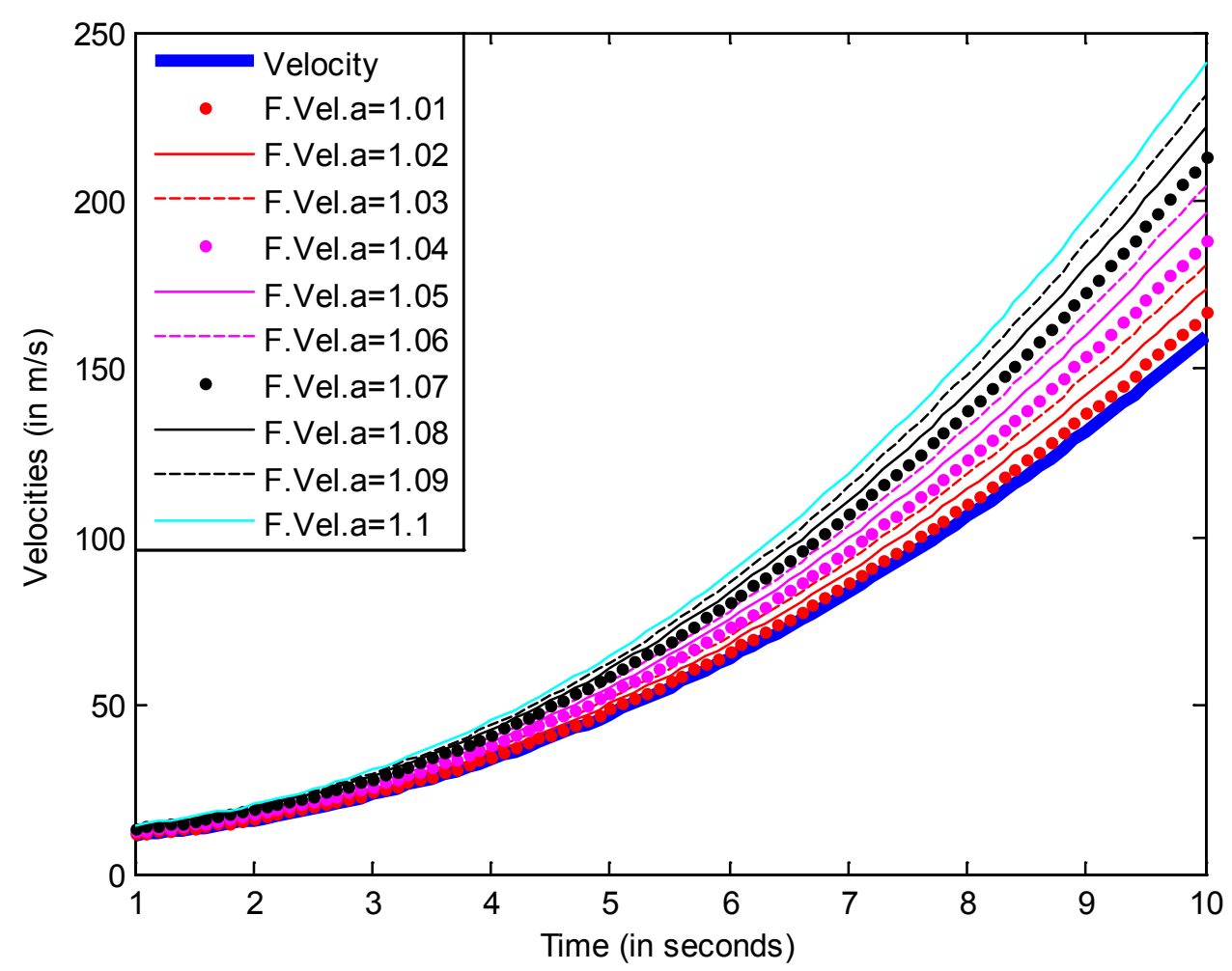

Figure 10. The fractional order derivatives of velocity for orders $=\{1.1,1.2,1.3,1.4,1.5,1.6,1.7,1.8,1.9,2\}$ (acceleration is variable).

\section{Conclusions}

The definition for fractional order derivative in $[14,15,16$, 17] was used in this study. It was seen that the order of FOD converges to 1 , the obtained result get similar to first derivative. When the order of FOD converges to 2, the obtained results are not similar or the same meaning with second derivative. The second derivative is the differentiation of first derivative and the order 2 of FOD means that the order of the first time derivation is 2 . These two terms are not same, and it was seen that acceleration is different from the FOD of order 2 . This case can be observed on the Figure 1, Figure 2, Figure 4, and Figure 6. The applied derivative operator is linear for order of 1 ; otherwise, the applied derivative operator is non-linear.

\section{REFERENCES}

[1] S. Das, "Functional Fractional Calculus", Springer-Verlag Berlin Heidelberg, 2011.

[2] A. Arikoğlu, İ. Özkol, "Solution of Fractional Differential Equations by Using Differential Transform Method", Chaos, Solitons and Fractals, vol.34, pp:1473-1781, 2007.

[3] O.P. Agrawal, "Analytical Schemes for a New Class of Fractional Differential Equations", Journal of Physics A: Mathematical andTheoretical, vol.40, pp:5469-5477, 2007.

[4] D. Baleanu, J.H. Asad, I. Petras, S. Elagan, A. Bilgen, "Fractional Euler-Lagrange Equation of Caldirola-Kanai
Oscillator", Romanian Reports in Physics, vol.64, pp:1171-1177, 2012.

[5] S. Chen,'Dark and Composite Rogue Waves in the Coupled Hirota Equations", Physics Letters A, vol.378, pp:2851-2856, 2014.

[6] E.F. El-Shamy, F.S. Gohman,'Propagation and Collision of Soliton Rings in Quantum Semiconductor Plasmas", Physics Letters A, vol.378, pp:2688-2694, 2014.

[7] J.-H. He, S.K. Elagan, Z.B. Li, “Geometrical Explanation of the Fractional Complex Transform and Derivative Chain Rule for Fractional Calculus", Physics Letters A, vol.376, pp:257-259, 2012.

[8] A. Alexopoulos, G.V. Weinberg, "Fractional-Order Formulation of Power-Law and Exponential Distributions", Physics Letters A, vol.378, pp:2478-2481, 2014.

[9] M.R. Ubriaco, "Entropies based on Fractional Calculus", Physics Letters A, vol.373, pp:2516-2519, 2009.

[10] R.W. İbrahim, J. Sokol, "On a New Class of Analytic Function Derived by a Fractional Differential Operator", Acta Mathematica Scientia, vol.34 (5), pp:1417-1426, 2014.

[11] Y. Yang, Y. Chen, "Convergence Analysis of the Jacobi Spectral-Collocation Method for Fractional Integro-Differential Equations", Acta Mathematica Scientia, vol.34 (3), pp.673-690, 2014.

[12] M. Stojanovic, " Regularity of Solutions to Nonlinear Time Fractional Differential Equations", Acta Mathematica Scientia, vol.33 (6), pp.1721-1735, 2013.

[13] N. Li, C. Wang,"New Existence Results of Positive Solution for a Class of Nonlinear Fractional Differential Equations", 
Acta Mathematica Scientia, vol.33 (3), pp.847-854, 2013.

[14] A. Karcı, "Kesirli Türev için Yapılan Tanımlamaların Eksiklikleri ve Yeni Yaklaşım", TOK-2013 Turkish Automatic Control National Meeting and Exhibition, Malatya, Turkey, 2013.

[15] A. Karc1, “A New Approach for Fractional Order Derivative and Its Applications", Universal Journal of Engineering Sciences, 1:110-117, 2013.
[16] A. Karc1, A. Karadoğan, "Fractional Order Derivative and Relationship between Derivative and Complex Functions", IECMSA-2013:2 $2^{\text {nd }}$ International Eurasian Conference on Mathematical Sciences and Applications, Sarajevo, Bosnia and Herzogovina, 2013.

[17] A. Karc1, A. Karadoğan, "Fractional Order Derivative and Relationship between Derivative and Complex Functions", Mathematical Sciences and Applications E-Notes, 2:44-54, 2014. 